\title{
Extended CNP Framework for the Dynamic Pickup and Delivery Problem Solving
}

\author{
Zoulel Kouki, Besma Fayech Chaar, Mekki Ksouri \\ National Engineering School of Tunis, Tunis, Belvedère. Tunisia \\ Email : zoulel.kouki@topnet.tn, \{Besma.fayechchaar, Mekki.ksouri\}@insat.rnu.tn
}

\begin{abstract}
In this paper, we investigate in the applicability of the Contract Net Protocol negotiation (CNP) in the field of the dynamic transportation. We address the optimization of the Dynamic Pickup and Delivery Problem with Time Windows also called DPDPTW. This problem is a variant of the Vehicle Routing Problem (VRP) that may be described as the problem of finding the least possible dispatching cost of requests concerning the picking of some quantity of goods from a pickup to a delivery location while most of the requests continuously occur during the day. The use of contract nets in dynamic and uncertain domains such as ours has been proved to be more fruitful than the use of centralized problem solving [9].We provide a new automated negotiation based on the CNP. Negotiation process is adjusted to deal intelligently with the uncertainty present in the concerned problem.
\end{abstract}

\section{Introduction}

In this paper, we deal with the DPDPTW problem which is NP-hard since it is a variant of the well-known NP-hard combinatorial optimization VRP [7]. It is made harder because of the real time requests occurrence and the mandatory precedence between the pick-up and the delivery customer locations [5], [8].

We propose a multi-agent based approach based on the CNP Negotiation. New requests assignment to vehicles will be done according to the rules of the CNP and vehicle agents are responsible for their own routing. Thus, proper pricing strategies are needed to help the system carrying out the minimum transportation and delay costs.

The remainder of the paper is structured as follows: In Section 2, we show a literature review illustrating uses of the CNP in the VRP variants solving. Section 3 gives a detailed description of the DPDPTW. The Extended CNP framework is then globally presented in section 4 . Section 5 and section 6 deal with the details of the insertion and optimization processes of the framework. In section 7 we discuss some implementation driven results. Final concluding remarks follow in Section 8 .

Please use the following format when citing this chapter:

Kouki, Z., Chaar, B.F. and Ksouri, M., 2009, in IFIP International Federation for Information Processing, Volume 296; Artificial Intelligence Applications and Innovations III; Eds. Iliadis, L., Vlahavas, I., Bramer, M.; (Boston: Springer), pp. 61-71. 


\section{Related Literature}

\subsection{Dynamic Vehicle Routing Problems}

Most techniques and models used in transportation planning, scheduling and routing use centralized approaches. Several techniques and parallel computation methods were also proposed, to solve models using the data that are known at a certain point in time, and to re-optimize as soon as new data become available [3] [7][8][12]. Psaraftis refers to the routing and scheduling in dynamic environments as if the output is not a set of routes, but rather a policy that prescribes how the routes should evolve as a function of those inputs evolving in real-time[7], [8].

\subsection{Agent Technology and Agent Based Transportation Planning}

Contracts are a powerful coordination mechanism in distributed systems. The CNP has been applied since about 1980. It was first introduced by smith [11] in order to deal with task distribution problems.

Recent researches are investigated in the applicability of multi-agent systems in the field of transportation control. Sandholm [9] applied the Transportation CNP system (TRACONET), a bidding protocol, where dispatch centres of different companies cooperate automatically to provide a least cost vehicle routing. TRACONET extended CNP with bidding and awarding decision processes based on marginal cost calculations. Fischer and al. (1996) developed MARS: a system for cooperative transportation scheduling and a simulation test bed for multi-agent transport planning. The Cooperative Information Agent (CIA) framework introduced the notion of obligations, which was broadened by Contractual Agent Societies to support the fluid organisation of agent societies.

\section{Problem Description}

\subsection{Notations}

In the DPDPTW, a set of customers call the dispatch center during the current day before a fixed call for service deadline, asking for the transportation of some load $q_{v}$ from a pick-up location $O_{v}$ to a delivery location $D_{v}$. These requests occurrence is considered as the single source of the problem's uncertainty. They are denoted by immediate requests and should be scheduled for the same day. 
The dispatch center has at its disposal $M$ vehicles moving at a desired fixed velocity and having a maximum capacity $Q$ which should never be exceeded. Each vehicle starts and ends its route $R_{k}$ at the central depot $v_{0}$ respectively at time $\underline{t_{k}}$ and $\overline{t_{k}}$. It starts its route with empty load $q_{k}(t=0)=0$.

Let $N$ be the set of transportation requests of cardinality $n$ and let $N^{+}=\underset{i \in N}{\cup} O_{i}$ and $N^{-}=\cup_{i \in N} D_{i}$ be the sets of the pick-up and delivery locations, respectively. For each location $v \in N=N^{+} \cup N^{-}$, relevant attributes concern mainly: the geographical location $\left(x_{v}, y_{v}\right)$, the on-site service time $\left(t_{s}\right)_{v}$ and the time window $\left[e_{v}, l_{v}\right]$, where $e_{v}$ and $l_{v}$ are respectively called release date and service deadline of the location $v$.

For each location $v \in N=N^{+} \cup N^{-} \cup\left\{v_{0}\right\}$, we consider an arrival time $\left(t_{A}\right)_{v}$, and departure time $\left(t_{D}\right)_{v},\left(t_{A}\right)_{v}$ and $\left(t_{D}\right)_{v}$ verify $\left(t_{A}\right)_{v}+\left(t_{s}\right)_{v} \leq\left(t_{D}\right)_{v}$

For each pair of locations $v_{i} \in V$ and $v_{j} \in V, t_{i j}$ is the travel cost between $v_{i}$ and $v_{j}$.

\subsection{Hypotheses}

- The vehicle is not allowed to skip its next service location, once it is travelling towards it [5][3] .

- We consider the "Wait First" waiting strategy. Once the pickup or delivery service of some location is finished, the vehicle should wait for its next departure time as long as it is feasible, so that it reaches its next destination after its time window starts [10].

\subsection{Constraints}

- The service should be made within the time window, and never begin before $e_{v}$. A penalty is incurred in the objective whenever $l_{v}$ is exceeded.

- The precedence constraint between the pick-up and the delivery should be respected.

\subsection{Optimization Criterion}

The objective is to minimize the transportation costs. The global cost function at time $\mathrm{t}$ is denoted by $C_{\text {DPDRTW }}(t)$ and can be written as in [5] [3]: 


$$
\sum_{0 \leq k \leq M} T_{k}+\alpha \sum_{v \in N}\left\{\max \left(0, t_{v}-l_{v}\right)\right\}+\beta \sum_{0 \leq k \leq M}\left\{\max \left(0, \overline{t_{k}}-l_{0}\right)\right\}
$$

$\alpha$ and $\beta$ are the weight parameters and $T_{k}$ is the travel time of $R_{k}$.

$\sum_{0 \leq k \leq M} T_{k}$ is the total travel time over all vehicles.

$\sum_{v \in N} \max \left\{0, t_{v}-l_{v}\right\}$ is the penalty, for violating the time window for all the custom-

ers of $V \backslash\left\{v_{0}\right\}=N^{+} \cup N^{-}$.

$\sum_{0 \leq k \leq M} \max \left\{0, \overline{t_{k}}-l_{0}\right\}$ is the sum of the overtime of all vehicles.

\section{Extended CNP Framewok for the DPDPTW Solving}

\subsection{Global Description of our Extended CNP Framework}

The main task of our model is to find the best possible solution to the DPDPTW. This is done by finding each time the best routing and scheduling of the set of available requests. We define routing as the act of determining an ordered sequence of locations on each vehicle route and scheduling as the act of determining arrival and departure times for each route location.

The dispatch center is represented by the dispatch agent who interacts with the vehicle agents representing the vehicles. Negotiation concerns either the insertion of the new requests or the optimizing of requests insertions into the planned routes.

In fact, the dispatch agent acts uniquely as the manager of new requests insertion negotiations, while vehicle agents may act either as managers of the optimizing negotiations or as participants of both types of negotiations. However, they may certainly not take both roles in the same time.

In the original CNP, several managers may be involved and announcements are allowed to be simultaneous. The available participants evaluate task announcements made by the managers and submit bids on those they think convenient, then managers evaluate the bids and contracts are awarded to the most appropriate bidders [11].

However, our model considers negotiations to be held one by one, because of the complicated nature of the addressed problem. The negotiation process effectively handles the real time events by going through only feasible solutions. Only vehicle agents that are effectively able to carry on the requests without exceeding the vehicles capacities and violating the customers' time windows constraints are involved, and bids are binding so that each vehicle is obliged to honour its awarded tasks. At the end of a negotiation, the task is awarded to the vehicle agent 
that had submitted the least price bid and who is responsible for improving the routing and scheduling of its not yet served locations.

In order to reduce the uncertainty involved by new requests incurrence, we assume that vehicle agents are completely aware of their environment. All the information about available unassigned requests is accessible to the vehicle agents.

A global description of our multi-agent system (MAS) behaviour is presented in figure2.

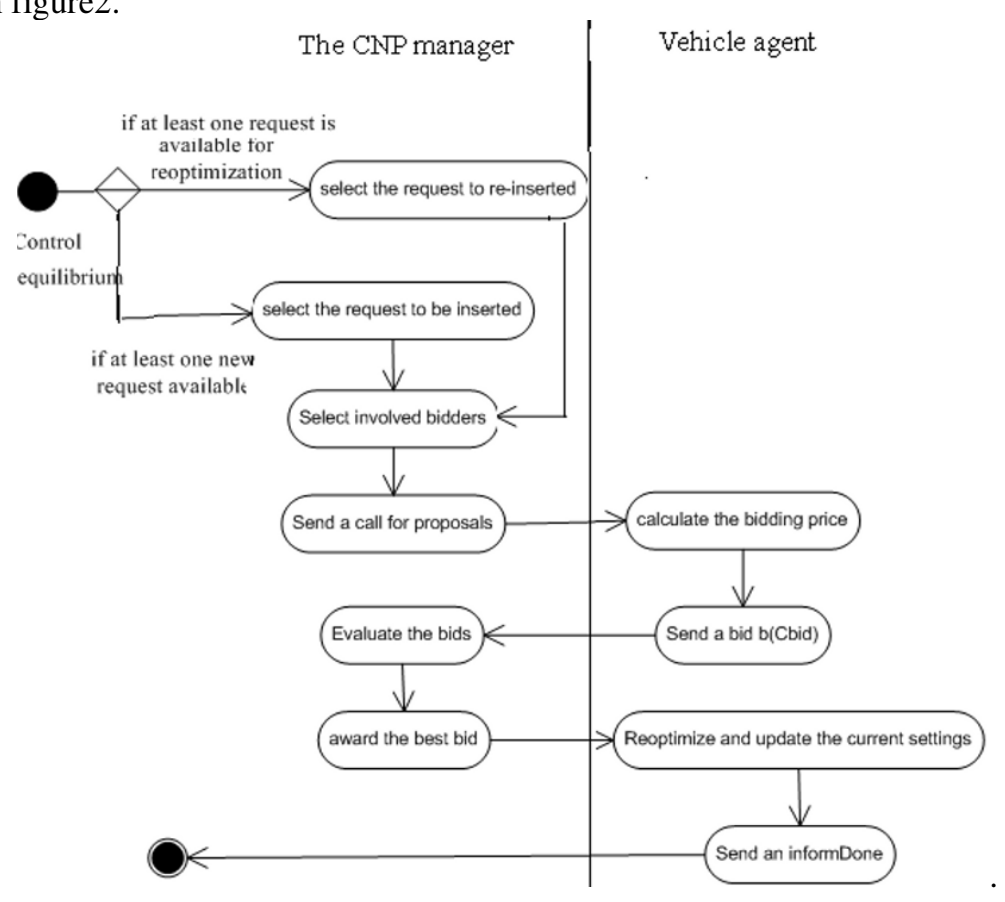

Fig 2. Global description of our DPDPTW- Extended CNP framework.

\subsection{Insertion Process}

We consider calls for service to arrive one-by-one and to be also announced one by one. The Dispatch agent is the manager of requests insertion tasks. While the set of available requests is not empty, the dispatch agent waits for the system to reach a global equilibrium and then establishes a new negotiation contract dealing with the most impending request. It issues a call for proposals act which specifies the task by giving all the details about the request, as well as the constraints and the conditions placed upon this task execution. Vehicle agents selected as potential participants to the negotiation receive the call for proposals. Their responses, referred to, as bids or submissions indicate the least possible price of inserting the request [2][4]. 


\subsection{Optimizing Process}

The second process aims to improve planned routes, it concerns the moving of requests from a first to a second route. This class of negotiations involves only Vehicle agents. Actually, in addition to bidding for new requests, each vehicle agent is also responsible for planning and scheduling its pickup and delivery services. Thus, vehicle agent may sell its own requests in order to reduce penalties put upon its objective function, to remove expensive requests or to be ready to accept some a coming request when its insertion seems to be more beneficial.

Moreover, in order to avoid visited solutions, vehicle agents use their feed back of last negotiations to make a good selection of the requests to be announced and of the vehicles to bid for the announced request.

\section{$5 \quad$ Insertion Process}

\subsection{Eligible Tasks and Bidders}

When at least one new request is available, the dispatch agent establishes a new contract to negotiate the request insertion possibilities with the interested vehicles. When several requests are available, they are sorted according to their pick up deadlines, and then announced one by one.

For each announcement, selection of bidders is based on the request insertion feasibility chances. The dispatch agent selects vehicles that are likely to offer feasible routes.

We assume that, each available request has at least one feasible insertion in the current routes, so that for each announcement, at least one vehicle is eligible for the negotiation. Eligible vehicles are those verifying both constraints of capacity and time window.

- Capacity constraint Check

For each eligible vehicle agent, there exists at least one pair of possible positions $i, j$ in the ordered sequence of service locations of the route $R_{k}$ such as $v^{+}$ may be inserted just next to $i$ and $v^{-}$next to $j$ : and that verify :

$$
\left.\operatorname{lt}_{D}\right)_{i} \leq t \leq\left(t_{D}\right)_{j}\left\{q^{k}(t)\right\} \leq Q-d_{v} .
$$

In fact the insertion of the request $v$ is possible only while (3) is maintained true during the period of time between the departure time $\left(t_{D}\right)_{i}$ from the $i^{\text {th }}$ location of the route towards the pickup location $v^{+}$and the departure time $\left(t_{D}\right)_{j}$ from the $j^{\text {th }}$ location of the route towards to the delivery location $v^{-}$.

- $\quad$ Time Window constraint check 
Let us divide the vehicle route into two portions: the first portion starts from time $t=0$ until the current time and the second portion starts from the current time and finishes at the time the vehicle ends its route, only the settings of the second portion of the route may be modified.

We define a possible routing block of a request $v$ in the route $R_{k}$ as a block that starts at the $i^{\text {th }}$ location and ends at the $j^{\text {th }}$ location of the route. i.e. $v^{+}$may be inserted just next to $i$ and $v^{-}$previous to $j$ : $i<\operatorname{position}(v+)<\operatorname{position}(v-)<j$

$i$ and $j$ should verify that insertion of $v^{+}$and $v^{-}$in the possible routing block bounded by $i$ and $j$ is a feasible insertion that respect $v^{+}$and $v^{-}$time windows constraints.

$$
\begin{aligned}
& e_{v+}+c \geq\left(t_{A}\right)_{i}+t_{v+, i} \geq e_{v+}-c \\
& l_{v-}-c \leq\left(t_{A}\right)_{j}-t_{v-, j} \leq l_{v-}+c
\end{aligned}
$$

$c$ is a parameter specified according to the overall minimum request time window and $t_{v+, j}$ is the travel cost between the pickup location $v^{+}$and the $j^{\text {th }}$ location of the route.

\subsection{One Contract at a Time}

In the original CNP, an agent could have multiple bids concerning different contracts pending concurrently in order to speed up the operation of the system [1], [11]. This was proved to be beneficial when the addressed tasks are independent and the price calculation processes are independent of the agent's assigned tasks and independent of any other tasks being negotiated at the same time.

However, in our model, negotiation concerns tasks that could be more or less inter-related and related with the already assigned tasks.

In fact, at a given time $t$, for each vehicle agent, because of the capacity constraint, the bidding decision depends conjointly on the vehicle's load which is the sum of its assigned locations loads, and the new request's load.

Besides, considering the problem's precedence and temporal constraints, some requests insertion may be considered to be prior to others. Locations of new and assigned requests may be close in space or in time and this makes their insertion into the same vehicle's route more beneficial. Then new requests pricing and bidding decisions are closely dependent of the earlier awarded requests.

Now, assuming that bids are binding, let us imagine that Vehicle agents may bid on several announcements at a time. In that case, tasks may become awarded to the vehicle while it is still bidding on other tasks. The

Its local settings changes should be considered by the bidder pricing functions. 
Otherwise the agent may submit wrong bids that it may be not able to honour and the resulting solution is unfeasible.

Considering all those reasons, we opt for the negotiations over only one contract at a time.

\subsection{Bidding and Pricing Mechanism}

The key issue to be discussed in this section is how to make pricing of requests insertions as accurate as possible. The pricing mechanism: a quasi-true valuation

We assume that the vehicle agent is bidding its true valuation such that the price of the task does not depend on the value other bidders attach to the task [].

However, because the problem is NP-hard, evaluating the cost of the announced request's insertion depends on the calculation of the truly optimizing function, which requires the search of an optimal routing and the calculation of departure and arrival times for every location included in the route, which is computationally demanding. Thus we consider a fast approximation $C_{a d d}(v)$ of the announced request $v$ adding cost to the routing solution. The vehicle agent determines first the possible routing block of the request, and while the bidding limit time is not reached it iterates calculating $C_{a d d}(v)$ for all the feasible pairs of positions $i, j$ included in the possible routing block and selects the best insertion.

$C_{a d d}(v)$ is the sum of the additional travel cost and the lateness eventually caused to customers expected to be served after the $i^{\text {th }}$ position:

- The additional travel cost is given by:

$$
\begin{gathered}
\left(c_{\text {add_travel }}\left(v^{+}\right)\right)_{k}=t_{i, v+}+t_{v+, i+1}-t_{i, i+1} \\
\left(c_{\text {add_travel }}\left(v^{-}\right)\right)_{k}=t_{j, v-}+t_{v-, j+1}-t_{j, j+1} \\
\left(c_{\text {add_travel }}(v)\right)_{k}=\left(c_{\text {add_travel }}\left(v^{+}\right)\right)_{k}+\left(c_{\text {add_travel }}\left(v^{-}\right)\right)_{k}
\end{gathered}
$$

-Penalties caused by time windows violations are given by:

$$
\begin{gathered}
\left(c_{\text {add_lateness }}(v)\right)_{k}=\sum_{p>i} \max \left(0, l_{p}-t_{A}(p)+\left(c_{\text {add_travel }}\left(v^{+}\right)\right)_{k}\right) \\
+\sum_{p>j} \max \left(0, l_{p}-t_{A}(p)+\left(c_{\text {add_travel }}\left(v^{-}\right)\right)_{k}\right)
\end{gathered}
$$

\section{4 Near Future Requests are Visible to Bidder Agents}

We assume that information about unassigned requests is visible to bidder agents. This information is used to intensify or reduce the agent desire for the acquisition of the negotiated request. 
This information about future is useful in situations like the following: Let us consider $v_{1}, v_{2}$ two disjoint requests. $v_{1}$ should be negotiated first, and $\left(c_{\text {add }}\left(v_{1}\right)\right)_{k}$ is the addition cost of $v_{1}$ into the route of the vehicle $k$.

It may exist $k, k^{\prime}, k^{\prime \prime} \in\{0, . ., M\} / k \neq k^{\prime}, k \neq k^{\prime \prime}\left(c_{\text {add }}\left(v_{1}\right)\right)_{k} \geq\left(c_{\text {add }}\left(v_{1}\right)\right)_{k^{\prime}}$

$\left(c_{\text {add }}\left(v_{1}\right)\right)_{k}+\left(c_{\text {add }}\left(v_{2}\right)\right)_{k} \leq\left(c_{\text {add }}\left(v_{1}\right)\right)_{k^{\prime}}+\left(c_{\text {add }}\left(v_{2}\right)\right)_{k^{\prime \prime}}$

This way, the $k^{\prime \text { th }}$ vehicle agent could be the winner of the request $\mathrm{v}$ while, awarding it to the vehicle $k^{\prime}$ would be more opportunistic.

\subsection{Requests Awarding}

Awarding a contract means assigning the service request to the successful bidder.

Request insertion is performed considering the solution proposed in the bid. Calculation of departure and arrival times of different locations is made immediately once the contract is awarded to the agent, according to the rules of the wait first waiting strategy, except for the possible routing block

The agent then uses its local optimization heuristics to make better the routing and scheduling of its assigned locations. Information about available requests is useful in the management of waiting times, in order to make possible and appropriate the insertion of coming requests.

\section{CNP Negotiation for Inter-routes Improvement}

Some assigned requests may result in large delays for services of the same vehicle.

Negotiations for post optimization are based on the idea of controlling cycles by avoiding the repetition of visited tours. Some moves or bids or announces or awards could have the status tabu, this helps avoiding the congestion of the negotiation network when an announcement is tabu for a bidder, the bidder is not eligible for this announcement.

In order to avoid infinite negotiation of post-optimization: an initiator could be tabu, in order to let the chance for others to sell their requests.

An agent may not announce a task, just acquired in the last negotiation. At least one change should be performed on its route to become authorized to announce the request.

When the request $v$ is announced, the vehicle that had it in the past in its route and that had announced it in the $\mathrm{n}^{\text {th }}$ negotiation is eligible for bidding on, only if at least one or more changes are performed into its route or in the current manger's 


\section{Experimental Results}

The purpose of the experiments was to validate the application of the CNP Multiagent negotiation in dynamic subjected to constraints domains

Our CNP based solution is implemented using the Jadex BDI agent-oriented reasoning engine realised as an extension to the widely used JADE middleware platform [14].

We used the test beds of Mitrovic-Minic [13]. We examined 10 instances of problems with one depot and respectively 100 and 500 requests. The service area is $60 * 60 \mathrm{~km}^{2}$, and vehicle speed is $60 \mathrm{~km} / \mathrm{h}$. In all instances, requests occur during the service period according to a continuous uniform distribution, and no requests are known in advance. The service period is 10 hours. Experiments were performed a simulation speed of 30 which means that one hour of real life operations is simulated in $1 / 30$ hours of computer time.

Preliminary experiments were performed to determine convenient selection and bidding parameter values.

At the end of experiments, we remarked that agents produce improved results. Considering future and past information made solutions to be more accurate. Table 1 provides the average experimental results performed with different initial fleet of vehicles. Tests considers ranging from five to twenty for the 100 requests instances and 20 to 40 for the 500 requests instances. when no near future or past information is considered, when only future information is considered and when both future and past information are considered.

Table 1. Experimental results

\begin{tabular}{lllllllll}
\hline & $\begin{array}{l}\text { SMinic WF Best } \\
\text { values }\end{array}$ & & $\begin{array}{l}\text { CNP with no } \\
\text { future nor past } \\
\text { information }\end{array}$ & $\begin{array}{l}\text { Extended CNP } \\
\text { with future } \\
\text { information }\end{array}$ & \multicolumn{2}{l}{$\begin{array}{l}\text { Extended CNP } \\
\text { with past and } \\
\text { future } \\
\text { information }\end{array}$} \\
\hline & Distance & $\mathrm{m}$ & Distance & $\mathrm{m}$ & Distance & $\mathrm{m}$ & Distance & $\mathrm{m}$ \\
\hline 100requests & 2453.61 & 13.76 & 2449.2 & 13.96 & 2462.3 & 13.42 & 2462.3 & 12.56 \\
\hline 500requests & 5874.11 & 25.95 & 5881.3 & 26.34 & 5872.4 & 25.88 & 5870.45 & 24.93 \\
\hline
\end{tabular}

The table reports total distance travelled and number of vehicles used (m).

\section{CONCLUSION}

We proposed a CNP automatic negotiation based approach for the DPDPTW optimization. Negotiation in our system concerned insertion of new requests and optimization of planned routes. It was real time and was adjusted in that way to deal with special features and constraints of the addressed problem. We considered the 
use of the one by one negotiation in order to carry out precise insertions .We considered also agents to be entirely cooperative since their objectives match that global system objective of reducing the dispatching costs.

We proposed agents to be well informed about their past negotiations and to use their knowledge about the tasks about to be negotiated.

Congestion of the negotiation network was avoided by the use of a thoughtful selection among tasks to be announced and bidders. Experimental results showed that CNP negotiations dealt successfully with dynamic problem of pick-up and delivery.

\section{REFERENCES}

1. Demazeau, Y. Multi-agents systems methodology. In Proceedings 4th Brazilian Symposium on Artificial Intelligence SIBA98, Porto Alegre. (1998)

2. FIPA. 1997 Specification. part 2, Agent Communication language, foundation for intelligent physical agents, Geneova, Switzerland. http://www.cselt.stet.it/ufv/leonardo/fipa/index.htm

3. Gendreau, M., Guertin, F., Potvin, J.-Y., and Séguin, R. 2006 GendreauNeighborhood search heuristics for a dynamic vehicle dispatching problem with pick-ups and deliveries. Transportation Research Part C 14, 157-174.

4. Guessoum, Z.. 2003 Modèles et Architectures d'agents et de systèmes Multi-agents adaptifs. Dossier d'habilitation de l'Université de Paris 6, France.

5. Larsen, A. 2001 The Dynamic Vehicle Routing Problem PhD thesis, IMM-Danemark.

6. Lund, K., Oli B. G. M, and Jens M. R. 1996 Vehicle Routing Problems with Varying Degrees of Dynamism. Technical report, IMM, The Department of Mathematical Modelling, Technical University of Denmark.

7. Psaraftis, H. N. 1988 Vehicle Routing: Methods and Studies, chapter Dynamic Vehicle Routing Problems, pages 223\{248. Elsevier Science Publishers B.V. (North Holland).

8. Psaraftis, H. N. 1995 Dynamic vehicle routing: Status and prospects. Ann. of Oper. Res., 61:143-164.

9. Sandholm, T. 1993. An Implementation of the Contract Net Protocol Based on Marginal Cost Calculations. In Proceedings 11th National Conference on Artificial Intelligence (AAAI).

10. Mitrovic-Minic, S., G. Laporte. 2004. Waiting strategies for the dynamic pickup and delivery problem with time windows. Transportation Res. $\mathrm{B}<\mathrm{b}>38</ \mathrm{b}>635-655$.

11. Smith, R.G. 1980. The Contract Net Protocol: High-Level Communication and Control in a Distributed Problem Solver. IEEE Trans. on Computers C-29(12):1104-1113.

12. Thierry, G., Didier, J., Dominique, F., Christian. A, and Élodie, C. Transport à la demande points à points en zone peu dense: Proposition d'une méthode d'optimisation de tournées.

13. http://www.fernunihagen.de/WINF/inhfrm/bench-mark_data.htm

14. http://vsis-www.informatik.unihamburg.de/pro-jects/jadex/ 